\title{
"DENTRO DAS CONDIÇÕES QUE A GENTE TEM": PERCEPÇÕES DE NUTRICIONISTAS SOBRE GASTRONOMIA EM HOSPITAL UNIVERSITÁRIO
}

\author{
“WITHIN OUR CONDITIONS”: PERCEPTIONS OF NUTRITIONISTS ABOUT \\ GASTRONOMY IN A UNIVERSITY HOSPITAL
}

\section{"DENTRO DE LAS CONDICIONES QUE NOSOTROS TIENE": PERCEPCIONES DE NUTRICIONISTAS SOBRE GASTRONOMÍA EN HOSPITAL UNIVERSITARIO}

\author{
Even Jheice Calixto Oliveira ${ }^{1}$, Tatiana Coura Olivera ${ }^{2}$, Virginia Souza Santos ${ }^{3}$
}

Como citar esse artigo: Oliveira EJC, Olivera TC, Santos VS. "Dentro das condições que a gente tem": Percepções de nutricionistas sobre gastronomia em hospital universitário. Rev Enferm Atenção Saúde, v. 9, n.02, 28-38, 2020. DOI: 10.18554/reas.v9i2.3966.

\section{RESUMO}

Objetivo: $\mathrm{O}$ presente trabalho propõe uma reflexão sobre sentidos atribuídos à gastronomia hospitalar, considerando a experiência de nutricionistas que atuam tanto na produção de refeições, quanto na clínica em um hospital universitário. Método: Partindo de uma perspectiva qualitativa, realizou-se entrevistas semiestruturadas com 15 nutricionistas. Resultados: A análise de conteúdo evidenciou categorias relacionadas ao custo da gastronomia frente aos subsequentes cortes orçamentários e a ressignificação do conceito. Conclusão: Desvelou-se uma nova perspectiva gastronômica que frente às adversidades financeiras, valorizou os hábitos dos pacientes e em sinergia com as necessidades dietoterápicas construiu uma alternativa possível pautada no diálogo entre equipe técnica e paciente.

Descritores: Alimentação Coletiva. Dieta; Nutricionistas; Hospitais; Humanização da Assistência.

\begin{abstract}
Objective: This paper proposes a reflection on the meanings attributed to hospital gastronomy, regarding the experience of nutritionists who work both in the production of meals and in the clinic at a university hospital. Method: From a qualitative perspective, semi-structured interviews were conducted with 15 nutritionists. Results: The content analysis showed categories related to the cost of gastronomy in face of the subsequent budget cuts and the resignification of the concept. Conclusion: A new gastronomic perspective was unveiled that, in the face of financial adversities, valued the habits of patients and in synergy with dietotherapic needs built a possible alternative based on the dialogue between the technical team and the patient.
\end{abstract}

Descriptors: Collective Feeding; Diet; Nutritionists; Hospitals; Humanization of Assistance.

\footnotetext{
${ }^{1}$ Nutricionista, Bacharela em Nutrição pela Universidade Federal de Viçosa Campus Rio Paranaíba, Rio Paranaíba - MG, jheicecalixto@gmail.com. Orcid: 0000-0003-1373-5981

${ }^{2}$ Nutricionista, Doutora em Ciências na área de Epidemiologia em Saúde Pública pela Escola Nacional de Saúde Pública Sérgio Arouca (FIOCRUZ), Universidade Federal de Viçosa, Campus Rio Paranaíba, Rio Paranaíba MG, tatiana.coura@ufv.br. Orcid: 0000-0001-6349-2522

${ }^{3}$ Nutricionista, Doutora em Atenção à Saúde pela Universidade Federal do Triângulo Mineiro, Universidade Federal de Viçosa, Campus Rio Paranaíba, Rio Paranaíba - MG, virginiasantos@ ufv.br Orcid: 0000-0002-66658097
} 


\section{RESUMEN}

Objetivo: Este trabajo propone una reflexión sobre los significados atribuidos a la gastronomía hospitalaria, considerando la experiencia de nutricionistas que trabajan tanto en la producción de comidas como en la clínica de un hospital universitario. Metodo: Desde una perspectiva cualitativa, se realizaron entrevistas semiestructuradas con 15 nutricionistas. Resultados: El análisis de contenido mostró categorías relacionadas con el costo de la gastronomía en vista de los recortes presupuestarios posteriores y la reformulación del concepto. Conclusiones: Se dio a conocer una nueva perspectiva gastronómica que, ante la adversidad financiera, valoró los hábitos de los pacientes y, en sinergia con las necesidades dietéticas, construyó una posible alternativa basada en el diálogo entre el equipo técnico y el paciente.

Descriptores: Alimentación Colectiva; Dieta; Nutricionistas; Los hospitales; Humanización de la Asistencia.

\section{INTRODUÇÃO}

A manutenção e/ou recuperação do estado nutricional no ambiente hospitalar é um desafio para os Serviços de Nutrição e Dietética. ${ }^{1}$ Parte desta realidade está ligada ao baixo índice de aceitação das refeições hospitalares, tendo em vista a atribuição de sentidos negativos, tais como insossa, sem gosto, fria, restritiva, dentre outros. ${ }^{2}$ Esta percepção pode estar ligada à necessidade dietoterápica de modificação física e/ou química das preparações, mas também às alterações fisiológicas no paladar dos pacientes, decorrentes do próprio tratamento farmacológico. ${ }^{3}$ A valorização de conceitos biológicos no âmbito da assistência nutricional em detrimento dos aspectos sensoriais e sentidos simbólicos da alimentação podem também se ligar à baixa aceitação da dieta, bem como a própria desnutrição intra-hospitalar. ${ }^{4-6}$

Numa tentativa de mudança desta perspectiva os serviços de alimentação hospitalares vêm implementando o conceito de gastronomia à rotina de produção. ${ }^{3}$ Tomando-se o mesmo como um conjunto de ações, treinamentos e desenvolvimento de técnicas de hotelaria voltadas à humanização das instituições de saúde ${ }^{4}$, na promoção de conforto aos pacientes durante sua recuperação.

Apesar dos benefícios descritos na literatura tais quais melhora significativa na aceitação das dietas e redução do resto ingestão, estudos brasileiros apontam o custo de sua implantação como principal desafio de sua execução nas instituições de saúde, principalmente nos públicos. ${ }^{5-7}$ Serviços hospitalares mistos implantam usualmente a gastronomia hospitalar (GH) no atendimento dos pacientes da rede privada $^{8}$ em detrimento aos da rede pública.

A implantação gastronomia hospitalar é, ainda hoje, um desafio tendo em vista que o serviço precisa fornecer a prescrição dietoterápica considerando micro, macronutrientes e consistência física numa refeição atrativa. Tendo em vista a 
importância do estado nutricional para a recuperação do paciente, bem como à complexidade de fatores ligados ao "comer" no âmbito hospitalar, o objetivo deste trabalho foi compreender os sentidos atribuídos à gastronomia a partir da perspectiva dos nutricionistas que atuam em um hospital público.

\section{MÉTODOS}

Trata-se de estudo de natureza qualitativa que buscou compreender, nos discursos sociais produzidos por nutricionistas, os diferentes sentidos atribuídos à GH. Realizado com profissionais do Serviço de Nutrição e Dietética de um hospital universitário na cidade de São Paulo - SP, a coleta de dados aconteceu no mês de janeiro de 2017, após aprovação do Comitê de Ética em Pesquisa (parecer consubstanciado $\mathrm{n}^{\circ} 1.741 .697$ ).

Desenvolvido a partir das seguintes etapas: (1) pesquisa teórica; (2) trabalho de campo e (3) análise e interpretação dos dados empíricos, a pesquisa teórica compreendeu uma revisão da literatura relacionada à temática, enquanto o trabalho de campo correspondeu à realização das entrevistas com as profissionais do setor. Por meio de um roteiro semiestruturado procedeu-se entrevistas com os profissionais, com duração aproximada de 30 minutos no próprio espaço hospitalar. As mesmas foram gravadas, codificadas (N01 a N15) para manter a confidencialidade e transcritas para posterior análise.

O roteiro direcionava a investigação para aspectos ligados à formação profissional e à relação de percepção sobre GH. Como apoio, utilizou-se um diário de campo, no qual eram registrados informações relevantes. Em relação à interpretação dos dados, optou-se pela análise de conteúdo (AC) considerando-se a perspectiva de Bardin $^{9}$, que buscou compreender as características e estruturas presentes no material transcrito. Realizouse num primeiro momento uma pré-análise do material: constituída de leitura e releitura do corpus tendo em vista a necessidade de organização dos achados do campo. Este momento permitiu a apreensão das estruturas de relevância e ideias centrais, que também foram se configurando como categorias empíricas: 1- os limites financeiros da aplicação da gastronomia e 2- uma ressignificação do conceito de gastronomia no contexto hospitalar.

Realizou-se posteriormente uma leitura horizontal e exaustiva das narrativas. Das transcrições, foram separadas partes semelhantes, para estabelecer conexões entre as categorias ou unidades de sentido. Posteriormente às análises de ordenação e classificação, seguiu-se para a tarefa de agrupamento das classificações, buscando compreender e interpretar o que foi exposto como mais relevante e representativo pelo 
grupo. Durante a interpretação dos achados foi necessário voltar ao referencial teórico, pois a relação entre os dados obtidos e a fundamentação teórica era imprescindível à interpretação.

\section{RESULTADOS E DISCUSSÃO}

\section{Caracterização do campo e dos participantes da pesquisa}

O serviço de Nutrição e Dietética (SND) desta unidade hospitalar realiza-se por meio da modalidade serviço próprio de gerenciamento, sendo a produção centralizada e a distribuição por meio de carros térmicos. $\mathrm{O}$ trabalho dos nutricionistas se divide entre as atividades realizadas nas áreas de nutrição clínica e de alimentação coletiva de forma a atender 258 leitos. A equipe do SND era composta por 15 nutricionistas, 7 cozinheiros, 57 auxiliares de cozinha e 4 auxiliares de serviços gerais, totalizando 83 colaboradores. Eram produzidas aproximadamente $\quad 1000 \quad$ grandes refeições/dia e distribuídas da seguinte forma: 300 no refeitório para funcionários do hospital, 580 para pacientes e 120 para acompanhantes. Também eram produzidos em média 95 lanches para os pais ou responsáveis por crianças no pronto socorro infantil e 300 fórmulas, água e leite materno para o serviço de pediatria.
Participaram do estudo todas as nutricionistas do SND $(\mathrm{n}=15)$. Todas as profissionais eram do sexo feminino, possuíam idade mediana de 52 anos (3559) e tempo de serviço de 24 anos (3-36). Em relação à graduação, houve um predomínio da realização no próprio estado de São Paulo. O tempo de formação mediano das profissionais foi de 29 anos (12-37).

Considerando-se o objeto de análise e o tempo mediano de formação das profissionais, é interessante ressaltar que faz pouco tempo que as matrizes curriculares de nutrição começaram a privilegiar conteúdos programáticos ligados à gastronomia. A própria inserção da disciplina na grade, mantém íntima relação com as transformações históricas que indicavam a atribuição de valor positivo à qualidade e a sofisticação na apresentação dos alimentos. Nesse sentido, muitos profissionais já inseridos no campo da alimentação coletiva sentiram necessidade de atender uma demanda de mercado, buscando formação na área. ${ }^{10-11}$

Cabe considerar que o SND da unidade em questão, apesar de público, vivenciou um período marcado por atividades que privilegiavam o aperfeiçoamento profissional dos nutricionistas e manipuladores de alimentos. Por meio da oferta de cursos de especialização na área de gastronomia e 
culinária, bem como o incentivo ao desenvolvimento de receitas, técnicas e aquisição de equipamentos para gestão do serviço. Foi um período de convergência de recursos financeiros e de ações institucionais: as memórias das participantes marcam este momento como um 'divisor de águas' em relação à aplicação da GH.

\section{O custo da gastronomia hospitalar:}

\section{dificuldade ou possibilidade criativa?}

A concepção do conceito de gastronomia está vinculada ao conceito de hotelaria $^{12}$, sendo considerada uma "vantagem competitiva" entre unidades que atendem o segmento. Para além desta questão, a implantação de técnicas gastronômicas promove uma maior aceitação da refeição servida, contribuindo para o adequado aporte nutricional ${ }^{13}$ e saúde dos pacientes.

Parte dos trabalhos que descrevem a GH revelam processos de elevado investimento de implantação e manutenção, considerando a substituição de embalagens descartáveis por louças e baixelas, uso de produtos mais sofisticados e de cortes e técnicas culinárias de maior complexidade, disposição de ingredientes decorativos e de pessoal para realizar empratamento e treinamentos. ${ }^{6,14-16}$ Mesmo entre as entrevistadas, percebe-se em uma ou outra narrativa a forte presença desta perspectiva:

\begin{abstract}
“A gente não consegue aqui ainda implantar o que eu conheço de gastronomia, o que eu tinha no outro serviço que eu trabalhava... Embora a gente tenha o cuidado de ter uma refeição variada, no conceito da gastronomia aqui, hoje em dia eu não vejo". (Participante N13)
\end{abstract}

Assim o custo apresenta-se uma dimensão importante e exige do profissional de Nutrição a capacidade de adaptação às condições adversas, principalmente decorrentes da crise financeira vivenciada pela população brasileira nos últimos anos. ${ }^{7}$ Diversos setores foram obrigados a rever e reestruturar seus serviços, o que incluía ajuste de custos com material e funcionários. ${ }^{6} \mathrm{O}$ próprio hospital, enquanto unidade vinculada à universidade pública, foi impactado e precisou neste novo contexto revisar as prioridades no âmbito da assistência à saúde.

\footnotetext{
"O que a gente tem, infelizmente, passado nesses últimos dois anos, é que (...) vem com um corte de gastos, então, a reitoria passa pro superintendente que passa pra cada divisão a necessidade de corte de gastos. A gente já passou em 2014 por [redução de] $20 \%$ dos nossos gastos, no ano passado [2015] mais $10 \%$, no ano passado [2016] mais 10\%; então, por mais que a gente queira, a gente tem que entrar no orçamento que (...) nos fornece... muita coisa a gente teve que mudar em relação ao cardápio, por causa justamente desses custos que a gente tem que tentar deixar disponível pra gente. Isso é uma coisa que diminui um pouco nossa possibilidade de variar algumas coisas, e adquirir coisas diferentes para os pacientes". (Participante N11)
} 
Nos discursos das entrevistadas a questão de redução de custos foi amplamente apontada como marcador do processo de transição do que "era" e do que "tornou a ser" o SND.

"Eu acho que a $\mathrm{GH}$ aqui já foi bem mais aplicada do que é agora, nisso que eu vejo que existe sim a aplicação mas, em função dos recursos isso foi um pouco interrompido: você pode melhorar a aparência das preparações”. (Participante N04)

O serviço aos pacientes é realizado em recipientes descartáveis que apresentam divisórias internas que separam as preparações quentes (acompanhamentos, prato principal e guarnição). As saladas são servidas em vasilhame separado, como aponta a fala da participante N05: "Mas a gente não tem condições de pôr num prato e a saladinha de outro jeito. E o caldinho de outro jeito. E a sobremesa de outro jeito, separados... A gente tem limitações nesse sentido".

A utilização de descartáveis é a alternativa mais plausível para a logística de distribuição, recolhimento e higienização dos itens, uma vez que o processo envolve não somente os custos com mão de obra, mas também as implicações ergonômicas para os copeiros, considerando algumas limitações para operações que envolvem objetos pesados como descreve a participante N06: "Teve muitas restrições nos últimos anos, por conta de verba, agora vai ter mais ainda, por conta de diminuição de funcionários, nutricionistas, então vai ficar mais complicado ainda aqui pra trabalhar."

$$
\text { Os ajustes orçamentários }
$$
relacionam-se aos sentidos atribuídos por algumas informantes à $\mathrm{GH}$, que relatam que a redução do custo unitário das refeições e da oferta de lanches não alterou o grau de satisfação dos pacientes, considerando os tópicos de qualidade avaliados pelo hospital.

\footnotetext{
"Eu participei de um trabalho sobre satisfação dos pacientes e eles sempre elogiaram muito. Uma, por conta do público que a gente atende, não é um público sofisticado, é um público de nível de médio pra baixo; não são tão exigentes e eles só gostam daquela comidinha mais trivial: arroz, feijão, e o que a gente serve foi muito bem avaliado." (Participante N06)

"Pelas últimas avaliações que têm sido feitas com os pacientes, tem sido boa, viu? E eu acho que até surpreende a gente... o pessoal tem falado bem, e assim, na verdade, não compromete a qualidade, é mais isso, não dá um up a mais no que pode ser oferecido." (Participante N10)
}

O empenho da equipe em servir refeições coloridas e harmoniosas preparadas a partir de ingredientes de baixo custo evidencia uma ação criativa para o enfrentamento dos desafios impostos pela crise econômica. Para além da variedade do cardápio e do uso de ervas aromáticas, novos sentidos são atribuídos à $\mathrm{GH}$, tal qual a garantia da segurança microbiológica e o 
respeito à cultura alimentar de grupos sociais específicos. ${ }^{5}$

“(...) mas assim, a gente procura incentivar novos sabores, o prato colorido, então eu vejo que o pessoal tem bastante preocupação na hora de montar o prato, o cardápio na refeição, de fazer o cardápio colorido, diversificado, as sopas nossas sempre tem três tipos de legumes, dois tipos... então procura ser variado o sabores e temperos também, se adequando mais ao gosto da população brasileira". (Participante N10)

"Considerando as nossas condições, comparando em geral, eu acho que tem coisa que a gente sempre fez aqui que é uma preocupação a mais. A gente utiliza bastante temperos, que é uma coisa que dá pra fazer, e eu sei que alguns hospitais públicos acabam não fazendo. Mas infelizmente, muito mais que isso a gente não consegue." (Participante N12)

\section{Ressignificação do conceito de gastronomia hospitalar: uma questão de}

\section{cuidado}

"Não vamos pensar em gastronomia em termos de sofisticação, vamos pensar quando a gente adapta as técnicas dietéticas/culinárias às necessidades do paciente, tanto em termos de aceitação como as exceções de dietoterapia que cada paciente tem. Fora a isso, eu acho que outro aspecto importante é você trazer o máximo de conforto pra esse paciente... a gente pensar que uma das poucas coisas que dão prazer pro paciente que está internado é a comida..." (Participante N14)

As narrativas evidenciam um olhar diferenciado no fornecimento da alimentação, para além do suprimento de nutrientes. A atribuição da importância do levantamento de informações sobre os hábitos e preferências alimentares dos pacientes para evitar inadequações na ingestão alimentar, reforça a percepção do 'comer' enquanto ato social, ligado à memórias e à própria identidade do sujeito, pelo qual perpassam hábitos e práticas culturais capazes de proporcionar prazer. ${ }^{17}$

\begin{abstract}
"Eu acho que os estudos e os treinamentos, não [é] só pros funcionários, mas também pros nutricionistas (...) e uma coisa que eu guardei e vi que isso é realmente uma coisa falha: que uma nutricionista de clínica, às vezes ela esquece que aquilo que tá no papel dele, e ele está pedindo pra prescrever pro paciente, é uma refeição, uma alimentação, são alimentos, é comida, um momento de conforto pra pessoa e tudo mais. E por outro lado, o nutricionista da produção esquece que ele é um profissional da saúde, e que ele tem que se preocupar também com essas opções: 'Ah, vamos trocar a margarina pelo azeite na refeição', ela não está pensando só na qualidade nutricional, está pensando na saúde. (...) Então essa questão da nutricionista da produção não dar atenção pra saúde, e a nutricionista de clínica lembrar que ela não tem que olhar só a saúde, mas tem que ver a refeição como uma parte boa, acho que isso é uma parte importante pra funcionar, aí sim, quando as pessoas estão cientes de tudo isso, elas vão realmente pensar em todo o resto, inclusive a alimentação, temperatura de administrar, o sabor, e tudo mais." (Participante N12)
\end{abstract}

A aceitação das refeições hospitalares emerge como quesito de avaliação da qualidade do serviço.

\footnotetext{
"A gente vê assim uma atividade que a gente faz muito com estagiários, equipe de aprimorandos, verificar a aceitação alimentar, porque é com isso que a gente vai ver a recuperação do paciente, a aceitação do nosso serviço, que acho que é o principal... a satisfação, e tudo mais... então isso é feito numa parceria com o pessoal da produção... a gente dá um retorno (...) então a gente procura assim... dar esse feedback pra ver como que está a aceitação.” (Participante N10)
} 
$\mathrm{Na}$ impossibilidade de serviços sofisticados, percebe-se um processo de ressignificação do conceito de GH: a equipe incorporou a dimensão do 'cuidado' e o respeito aos hábitos alimentares dos pacientes aos aspectos da gastronomia. Para Hartwell et al. ${ }^{18}$, considerando a experiência da refeição hospitalar, em ordem decrescente de importância estão os fatores sociais, os pessoais e os situacionais, reforçam ainda que a melhoria da qualidade da comida e a eficiência com que ela chega aos pacientes devem ser os objetivos mais importantes do serviço de alimentação hospitalar.

"Nós temos um paciente que não é elitizado, de um hospital particular, que tá acostumado com uma hotelaria. Então, dentro do nosso público-alvo, eles têm aceitado, porque nossa comida normalmente é bem caseira, mais próximo daquilo que eles fazem em casa. E o que eu percebo é que o nosso paciente gosta é da comida caseira... É a proximidade do dia a dia dele, e não ficar inventando coisas muito diferentes que ele não conhece, é você aproximar, com aquilo que ele comeria em casa." (Participante N11)

As percepções das entrevistadas apresentam pequenas divergências, que apesar de apontarem subjetividades, não produzem incongruências em si, pelo contrário, evidenciam aspectos por vezes desconsiderados no âmbito da pesquisa em $\mathrm{GH}$, tal qual a busca da qualidade, mesmo em gêneros simples e de baixo custo.

“O que eu preciso fazer lá atrás pra que a gastronomia que a gente consegue fazer, que não é muito grande, dê certo: eu tenho que servir uma coisa que esteja com boa qualidade". (Participante N01)

"Dentro das condições que a gente tem, a gente sempre procura assim, cada dia a gente faz um molho diferente, pra não ficar o cardápio [menos] variado; existe um cardápio rotativo mensal, então a cada mês esse cardápio roda e a gente vai tentando no dia a dia, de repente aprimorar ali um molho, ou fazer testes com algum temperinho novo, principalmente para aqueles pacientes que comem sem sal, então a gente coloca uma ervinha no arroz, faz um temperinho diferente, deixa um limãozinho, faz um vinagrete sem sal, pra ver se o paciente aceita melhor, então a gente vai tentando ali, adequar o melhor que a gente consegue". (Participante N11)

Para as informantes a perspectiva predominante do conceito de $\mathrm{GH}$ pode ser insuficiente caso não se dedique tempo à atenção nutricional tendo em vista que a aceitação da refeição hospitalar sofre influência dos sintomas e interferências orgânicas decorrentes das doenças, tratamento farmacológico e dos aspectos emocionais. $^{19}$

\footnotetext{
"Acho que têm muitos fatores que interferem. É diferente quando você está tomando 5 medicamentos diferentes e ter que comer. Um exemplo bem claro disso são os pacientes de ortopedia. Às vezes internam o motoqueiro, que sofreu um acidente. $\mathrm{O}$ cara comia muito bem em casa, chega aqui e não come nada: não é por causa da comida do hospital; ele tá com antibiótico, ele está com antiinflamatório, tá com protetor gástrico, ele tá acamado, ele tá com dor, então às vezes não é a comida que é o fator, ele não come não é porque ele não gostou, é porque realmente, naquele momento, não tem condições de comer". (Participante N05)
}

A gastronomia deve estar para além da aplicação de técnicas ou do uso de 
louças. Ela deve incluir a promoção do bem-estar do indivíduo e considerá-lo importante no processo de recuperação.

Taldivo e $\operatorname{Santos}^{20}$ elencam ainda que a aceitação das refeições hospitalares está relacionada ao oferecimento de preparações, que ao substituir ingredientes para atender às restrições inerentes às patologias, continuem saborosas e possuam adequado aspecto visual. Os autores destacam que refeições caseiras, semelhantes às que os pacientes possuem nas suas casas propiciam maior acolhimento.

"Então, tem uma dieta geral prescrita, e ele não tá conseguindo, então eu vou lá e ofereço mudanças. "Tá... E se ao invés do arroz e feijão a gente te mandar um macarrão? E se em vez de uma carne eu te mandar o frango? E se em vez de eu te dar comida normal, eu te der uma sopa hoje? Quem sabe você faz a digestão mais tranquilo? Posso mudar sua sobremesa? Você prefere uma gelatina, ao invés de uma fruta? Então, a gente tem autonomia pra fazer isso. E a gente procura fazer... A gente vai até o nosso limite." (Participante N05)

A ação do nutricionista deve ultrapassar a realização da triagem e da classificação do risco nutricional e englobar também a avaliação dos hábitos alimentares e a promoção da ingestão das refeições. Neste aspecto, as dimensões que emergiram das narrativas reforçam a necessidade de redirecionamento da atenção nutricional aos pacientes para além da sofisticação do serviço. Ou seja, a preocupação com o valor nutricional ou com o custo das preparações não podem se sobrepor ao cuidado com o paciente. Os profissionais devem manter atenção constante para a produção de refeições atrativas que se aproximem dos hábitos alimentares dos comensais. Caso contrário corre-se efetivamente o risco de que a alimentação não cumpra seu papel biopsicossocial.

\section{CONCLUSÕES}

A partir da análise das entrevistas, encontrou-se uma ressignificação no conceito de GH decorrente de adaptações econômicas enfrentadas continuamente. Embora o emprego da gastronomia, seja em alguns casos considerado uma prática onerosa, é possível aplicá-la em hospitais públicos. As técnicas gastronômicas são importantes e contribuem para a mudança no conceito estigmatizado de "comida de hospital", entretanto a GH não se restringe a elas. A experiência das profissionais aponta uma maior aproximação do serviço com o paciente e a adaptação aos seus hábitos e práticas alimentares, de forma que o cuidado na atenção nutricional respeite desejos e preferências, e o valorize em sua totalidade, considerando a cultura, bem como o quadro clínico. Esta percepção da $\mathrm{GH}$ pode fornecer um caminho mais criativo de enfrentamento das adversidades financeiras que se abatem sobre o segmento da alimentação coletiva, principalmente no âmbito do serviço público. Destaca-se, 
como limitação do estudo, sua realização em única unidade hospitalar. Salienta-se a necessidade de extrapolar a análise para outros estados e também para hospitais da rede privada.

\section{REFERÊNCIAS}

1. Toledo DC, Piovacari SMF, Horie LM, Matos LBN, Castro MG, Ceniccola GD et al. Campanha "Diga não à desnutrição": 11 passos importantes para combater a desnutrição hospitalar. Braspen J. 2018; 33(1):86-100.

2. Demário RL, Sousa AA, Salles RK. Comida de hospital: percepções de pacientes em um hospital público com proposta de atendimento humanizado. Ciênc Saúde Colet. 2010; 15(1):127582.

3. Ribas SA, Pinto EO, Rodrigues CB. Determinantes do grau de aceitabilidade da dieta hospitalar: ferramentas para a prática clínica? Demetra (Rio J). 2013; 8(2):137-48.

4. Martins P, Baratto I. Gastronomia hospitalar: treinamento em bases de cozinha. Revista Brasileira de Obesidade, Nutrição e Emagrecimento. 2018; 12(69):110-17.

5. Santos VS, Gontijo MC, Almeida MEF. Efeito da gastronomia na aceitabilidade de dietas hospitalares. Nutr Clín Diet Hosp. 2017; 37(3):1722.

6. Navarro DA, Boaz M, Krause I, Elis A, Chernov K, Giabra M, et al. Improved meal presentation increases food intake and decreases readmission rate in hospitalized patients. Clin Nutr. 2016; 35(5):1-6.

7. Mccray S, Maunder K, Barsha L, Mackenzie-Shalders K. Room service in a public hospital improves nutritional intake and increases patient satisfaction while decreasing food waste and cost. J Hum Nutr Diet. 2018; 31(6):734-8.

8. Parisenti J, Firmino CC, Gomes CE. Avaliação de sobras de alimentos em unidade produtora de refeições hospitalares e efeitos da implantação do sistema de hotelaria. Alim Nutr. 2008; 19(2):191-94.

9. Bardin, L. Análise de conteúdo. São Paulo: Edições 70; 2011.

10. Soares NT, Cavalcanti AA. Diretrizes curriculares nacionais para os cursos de nutrição: avanços, lacunas, ambiguidades e perspectivas. Rev Nutr. 2010; 23(5): 895-905.

11. Edelstein S. Molecular gastronomy transforms medical nutrition therapy for dysphagia. J Acad Nutr Diet. 2013; 113(9):31.

12. Taldivo BP, Santos MCT. Gastronomia hospitalar. Rev Conexão Eletrônica. 2016; 13(1):349-56.

13. Novinski APF, Araújo GC, Baratto I. Resto ingesta em uma unidade de alimentação e nutrição hospitalar na cidade de Pato Branco - PR. Revista Brasileira de Obesidade, Nutrição e Emagrecimento. 2017; 11(66):451-58.

14. Pinto CC, Alves, EA. A gastronomia no contexrto da hotelaria hospitalar: um estudo de caso na cidade do Rio de Janeiro, RJ, Brasil. Revista de Administração Hospitalar e Inovação em Saúde. 2017; 14(2):01-13.

15. Gonçalves MPM, Anjos JRC, Celemi LG, Gonçalves RD, Balderamas HA. A importância da gastronomia para a nutrição. Revista Empreenda Unitoledo. 2018; 2(1):251-63.

16. Castro ACL, Zanella CP. Conhecimentos e práticas de nutricionistas em gastronomia hospitalar. Nutrivisa. 2017; 4(1):32-8.

17. Canesqui AM, Garcia RWD. Antropologia e nutrição: um diálogo possível. Rio de Janeiro: Fiocruz; 2005.

18. Hartwell HJ, Shepherd PA, Edwards JSA, Johns N. What do patients value 
in the hospital meal experience?

Appetite. 2016; 96:293-98.

19. Randler C, Desch IH, Kampe VO,

Wüst-Ackermanna P, Wildec M,

Prokopd P. Anxiety, disgust and

negative emotions influence food

intake in humans. Int J Gastron Food

Sci. 2017; 7(1):11-15.

20. Taldivo BP, Santos MCT. Gastronomia

Hospitalar. Rev Conexão Eletrônica.

2016; 13(1):1-8.

RECEBIDO: 10/09/2019

APROVADO: $28 / 07 / 2020$

PUBLICADO: $12 / 2020$ 